\title{
O conceito de responsividade na Terapia Analítico Comportamental e suas implicações para a pesquisa de processo-resultado
}

\author{
The concept of responsiveness in Behavioral Analytical Therapy and its \\ implications on process-outcome research \\ El concepto de responsividad en la Terapia Analítico Conductual y \\ implicaciones para la investigación de proceso-resultado
}

Priscila Ferreira de Carvalho Kanamota ${ }^{1}$, Ana Carmen F. Oliveira ${ }^{2}$, Walquiria Lino da Silva Morais ${ }^{3}$, Denis Roberto Zamignani ${ }^{4}$, Roberta Bianca Marcelino de Almeida ${ }^{5}$, Anna Carolina Ramos ${ }^{6}$, Ananda Pantet ${ }^{7}$, Aline David de Oliveira $^{8}$, Lucas Murta de Andrade ${ }^{9}$, Natasha Hayamizu ${ }^{10}$, Bernardo Dutra Rodrigues ${ }^{11}$

[1] Faculdade Aldete Maria Alves - FAMA, Iturama/MG, Paradigma - Centro de Ciências e Tecnologia do Comportamento [2] [3] [4] [6] [7] [8] [9] [10] [11] [12] Paradigma - Centro de Ciências e Tecnologia do Comportamento [6] Paradigma - Centro de Ciências e Tecnologia do Comportamento, Departamento de Psicologia, Universidade Federal do Tocantins (UFT), Palmas/TO I Título abreviado: Conceito de responsividade na TAC e implicações para pesquisa I Endereço para correspondência: I Email: prifcarvalho@gmail denis@paradigmaac.org I doi: 10-18761/PAC.TAC.2019.016

Resumo: A clínica psicoterápica apresenta diversas demandas ao terapeuta, que vão desde as questões teóricas, relacionadas à abordagem adotada pelo profissional, até aquelas relacionadas ao cliente - suas características pessoais, demandas clínicas, expectativas com relação ao tratamento etc. Identificar como e quando responder a todas essas demandas é um desafio para o psicólogo, que deve ser responsivo às diferentes necessidades do caso clínico, visando a melhora do cliente. O presente artigo tem como objetivo conceituar o termo responsividade com base na visão analítico-comportamental e apresentar alguns aspectos relevantes para a sua consideração na produção de pesquisas no contexto clínico. Para isso, é apresentada a origem do termo, as definições do conceito de acordo com diferentes abordagens teóricas, o entendimento do conceito com base na perspectiva analítico-comportamental e sua importância para a pesquisa em psicoterapia.

Palavras-chave: responsividade; análise do comportamento; psicoterapia analítico-comportamental; pesquisa em psicoterapia. 
Abstract: Clinical practice in psychology presents several demands on the clinician, ranging from theoretical issues, related to the approach adopted by the professional, to those related to the client's - their personal aspects, therapeutic problems, expectations regarding the treatment, etc. Identifying how and when to respond to all these demands is a challenge for the clinical psychologist, who must be aware on being responsive to the client aiming therapeutic progress. The present article aims to conceptualize the term responsiveness based on the Behavior Analysis science and present some relevant aspects concerning the clinical research. Thus, the origin of the term responsiveness is presented, following by their definitions according to different theoretical approaches. A behavioral analytical definition of responsiveness and its importance for research in psychotherapy is suggested at the end of this article.

Keywords: responsiveness; behavior analysis; behavior therapy; psychotherapy research.

Resumen: La psicoterapia clínica presenta al terapeuta una serie de demandas, las cuestiones teóricas, relacionadas con el enfoque adoptado por el profesional, hasta las del cliente - sus características personales, problemas terapéuticos, expectativas con respecto al tratamiento, etc. Identificar cómo y cuándo responder a todas estas demandas es un reto para el psicólogo clínico, que debe ser responsivo a las diferentes necesidades del caso clínico, buscando la mejora del cliente. El presente artículo pretende conceptualizar el término responsividad desde el punto de vista del Análisis Conductual y presentar algunos aspectos relevantes para su consideración en contextos de investigación clínica. Para lograr este objetivo, presentase el origen del término, sus definiciones de acuerdo con diferentes enfoques teóricos, su comprensión desde una perspectiva del análisis conductual y su importancia para la investigación en psicoterapia.

Palabras-clave: responsividad; análisis conductual; psicoterapia analítico-conductual; investigaciones en psicoterapia. 
A Responsividade tem sido discutida como aspecto fundamental para o desenvolvimento de uma relação terapêutica de qualidade. O contexto do atendimento psicoterápico apresenta ao clínico inúmeras demandas: as teóricas (advindas da abordagem de atuação), as técnicas (instrumentos e métodos utilizados no atendimento), as do terapeuta (advindas do repertório de habilidades pessoais), as dos clientes (que inclui desde suas demandas clínicas, padrão de respostas mais frequentes, e expectativas para com o tratamento). Conseguir responder a todas essas demandas ou saber a quais delas responder em determinado momento se constitui um desafio para o clínico.

No contexto da pesquisa em psicoterapia, especialmente em estudos que envolvam algum tipo de manipulação de variáveis na intervenção, a responsividade terapêutica também consiste em um desafio. Isso porque, para o desenvolvimento de estudos com maior validade ecológica, o delineamento deve levar em consideração o contexto imediato do processo terapêutico nas diferentes fases do estudo (Stiles, 1999); Stiles, Honos-Webb \& Surko, 1998). O presente artigo visa apresentar uma conceituação analítico-comportamental do termo e levantar algumas implicações para sua consideração no âmbito da produção de conhecimento no contexto da clínica.

\section{A Origem do Termo Responsividade}

De acordo com o dicionário, responsividade tem a ver com quem responde e é a capacidade de dar uma resposta rápida e adequada à situação (Dicionário Infopédia, 2019).

Na psicologia, o termo responsividade remete a uma abordagem etológica da psicologia do desenvolvimento, mais propriamente aos estudos sobre a interação cuidador-criança e os padrões de apego (Ribas e Moura, 2004). Para teóricos do desenvolvimento, a responsividade parental é o elemento central na compreensão do desenvolvimento infantil e está ligada a teoria do apego (Bornstein, 1995; Ribas \& Moura, 2004; Ribas, Seidl de Moura, \& Ribas, 2003).

O apego é um vínculo recíproco e duradouro entre o bebê e o cuidador e tem um valor adaptati- vo para o bebê, assegurando que suas necessidades, tanto psicossociais quanto físicas sejam satisfeitas (Bowlby, 1984a; 1984b; MacDonald, 1998; Papalia \& Feldman, 2013). A qualidade deste vínculo, por sua vez, dependerá do fator responsividade (Bowlby, 1984a; Bretherton, 1992; Ribas \& Moura, 2004). Para Bowlby (1994a), o apego seguro depende da responsividade contingente dos pais em relação ao bebê, ou seja, da capacidade do adulto em mostrar-se sensível às pistas do bebê e responder nos momentos adequados com o sorriso, a fala etc. (Papalia \& Feldman, 2013).

Bretherton (1992) considerou que a responsividade materna possuía duas dimensões: 1) Afetiva (calor, proximidade, intimidade) e 2) Temporal (contiguidade). Sendo assim, a responsividade pode ser definida na área do desenvolvimento como o comportamento do cuidador de responder de forma afetuosa e rápida às necessidades dos filhos, de modo a promover a autonomia e autoafirmação na criança por meio do apoio emocional e da comunicação.

Responsividade é uma propriedade do comportamento, que pressupõe uma finalidade (Bretherton, 1992; Maccoby \& Martin, 1983). Diante de tal definição, o analista do comportamento tem a necessidade de operacionalizar tanto as variáveis envolvidas nos seus componentes afetivo e temporal, como na pressuposição de uma consequência final positiva.

\section{Por que Atentar Para a Responsividade na Terapia?}

É consenso que a psicoterapia se refere a uma forma especial de interação interpessoal (Norcross \& Wampold, 2011). A intervenção terapêutica consiste basicamente na interação entre dois indivíduos: o cliente - aquele que se encontra em algum tipo de sofrimento e busca ajuda para a melhoria das contingências de sua vida - e o terapeuta - aquele que dispõe de estratégias de análises e intervenções capazes de possibilitar a diminuição do sofrimento e aumento de contingências reforçadoras do cliente (Meyer \& Vermes, 2001; Santos, Santos, \& Marchezini-Cunha, 2012; Skinner, 2003; Vermes, Zamignani, \& Kovac, 2007). Dessa forma, estudar 
o processo psicoterápico envolve analisar as características comportamentais do terapeuta, as características do cliente, a interação entre elas, além das técnicas e estratégias utilizadas na intervenção.

As características comportamentais do terapeuta que compõem o processo terapêutico são geralmente denominadas na literatura como parte dos fatores comuns (fatores que influenciam no resultado do processo, independentemente da técnica ou abordagem terapêutica), referindo-se a comportamentos do terapeuta tais como empatia, calor humano, apoio, autenticidade, diretividade, entre outros (Sousa, 2011). Em relação às características do comportamento do cliente, a literatura aborda a complexidade inicial do problema enfrentado, o repertório comportamental, como resistência, impulsividade, passividade, seguimento ou não de regras e déficits em habilidades sociais, para citar algumas (Oshiro, 2011). Tais características do terapeuta e do cliente associadas aos chamados fatores específicos - que se referem à estrutura do tratamento, contrato, recursos e técnicas utilizadas em terapia - participam do processo de mudança do comportamento do cliente em terapia e podem facilitar ou dificultar tal processo (Castonguay \& Beutler, 2006; Meyer, 2001; Meyer \& Vermes, 2001). A essas complexas e dinâmicas relações que acontecem entre o terapeuta e o cliente dá-se o nome interação terapeuta-cliente (Zamignani \& Meyer, 2007, 2011).

Dada a singularidade e a relevância dessas interações, Silveira (2000) defende que esse relacionamento só deve ser denominado Terapêutico quando proporcionar o alcance das metas terapêuticas. A interação terapeuta-cliente ao longo das sessões psicoterápicas pode estabelecer um cenário no qual novos comportamentos possam ser modelados proporcionando ganhos terapêuticos para o cliente (Follette, Naugle, \& Callaghan, 1996; Kohlenberg \& Tsai, 2001/1991).

Ao esmiuçar os processos envolvidos na interação entre o terapeuta e o cliente, é possível encontrar: (1) o referencial teórico conceitual que embasa a intervenção, (2) as características interpessoais do cliente, (3) as características interpessoais do terapeuta, (4) as habilidades terapêuticas, (5) as técnicas e recursos utilizados no atendimento e (6) a etapa ou momento histórico da sessão. Cada aspecto envolvido na interação já foi estudado em pesquisas com resultados que apontam para uma correlação ou até relação com a melhora terapêutica do cliente (Amaral, 2010; Elliott, Bohart, Watson, \& Greenberg, 2011; Kanamota, Bolsoni-Silva, \& Kanamota, 2016; Oshiro, 2011; Oshiro, Kanter \& Meyer, 2012; Pinto, 2007; Silveira, 1997; Zamignani, 2007).

As habilidades terapêuticas estão relacionadas tanto a aspectos positivos do processo - estabelecimento do vínculo terapêutico, diminuição da resistência do cliente e resultados satisfatórios (Kanamota et al., 2016; Elliot, Bohart, Watson \& Greenberg, 2011; Kivlighan, Angelone, \& Swafford, 1991; Meyer, Del Prette, Zamignani, Banaco, Neno, \& Tourinho, 2010; Meyer \& Vermes, 2001; Orlinsky, Grawe, \& Parks, 1994; Rocha, 2011) quanto a ganhos terapêuticos pouco expressivos ou até mesmo ao abandono do processo psicoterapêutico. Tomando como exemplo a empatia, se esta for utilizada excessivamente em sessões iniciais, sem ser acompanhada por outros tipos de intervenção, tende a proporcionar pouco efeito terapêutico (Kanamota et al., 2016; Harwood, 2003; Patterson \& Chamberlain, 1994). Intervenções mais diretivas (como recomendação ou orientação), por sua vez, estão relacionadas a prejuízos no processo psicoterapêutico, tais como o aumento de comportamentos de resistência por parte do cliente e dificuldade no estabelecimento de relações entre variáveis, (Kanamota et al., 2016) mas também relacionadas a resultados favoráveis, por exemplo, quando utilizada em casos de clientes depressivos, resistentes e/ ou associadas a comportamentos como aprovação (Bachelor \& Horvath, 1999; Harwood \& Eyberg, 2004; Orlinsky et al., 1994; Silveira, 2000; Silveira, Bolsoni-Silva \& Meyer, 2010).

Embora as pesquisas sigam desvendando aspectos da interação terapeuta-cliente que se relacionem com a efetividade terapêutica, descrevendo o como e quando fazer, o que se espera do terapeuta continua sendo a chave para o processo em que o cliente se sinta de fato compreendido e atendido em suas reais necessidades. Nesse sentido, o estudo da responsividade na interação terapeuta-cliente pode lançar luz a intervenções bem-sucedidas, outrora atribuídas ao feeling dos terapeutas experientes.

As definições do conceito de responsividade terapêutica variam com o passar do tempo e de acordo com a abordagem teórica. Na década de 1970 discu- 
tia-se a concepção de que a psicoterapia se desenvolve sob condições relacionais, pelas quais o terapeuta é orientado a responder às necessidades e problemas do cliente, a partir de dois conceitos das teorias psicanalíticas e da psicologia do self: frustração ótima e gratificação ideal. Na relação cuidador-bebê, a frustração ótima é aquela experienciada pela criança, branda o suficiente a ponto de não ser traumática e intensa o suficiente para que seja significativa. Para Kohut (1989/1971, p. 185) "O analista especialista ajudará o paciente mantendo esse desconforto dentro de limites toleráveis; isto é, conduzirá a análise de acordo com o princípio da frustração ótima ${ }^{1 "}$ para que ocorra o adequado desenvolvimento do self. Na mesma perspectiva, o processo de terapia deve oferecer também um nível de gratificação ideal, entendido como a satisfação de algumas necessidades e desejos do cliente. A orientação é que o "o terapeuta não deveria dar nem muito nem pouco, mas o suficiente em um momento específico do processo terapêutico, a fim de permitir a frustração ótima e, consequentemente, o crescimento psicológico do cliente $^{2 ”}$ (Sousa, 2011, p. 19).

Já na década de 1980, Bacal defendeu que os conceitos de frustração ótima e gratificação ideal deviam ser expandidos para a ideia mais abrangente da responsividade ótima. Nas palavras de Bacal:

\footnotetext{
"Responsividade ótima é definida como a responsividade do analista que é terapeuticamente mais relevante em dado momento no contexto de um paciente em particular e sua doença. A empatia ou introspecção vicária é o processo pelo qual o terapeuta passa a entender o paciente, transformando-se em seu mundo interior. A responsividade ótima, por outro lado, refere-se ao terapeuta comunicar sua compreensão ao paciente"3 (Bacal, 1985, p. 202).
}

1 [El analista experto ayudará al paciente manteniendo este malestar dentro de límites tolerables; o sea, conducirá el análisis según el principio de frustración óptima.]

2 [In this sense, the therapist should not give too much or too less, but the sufficient in a specific moment of the therapeutic process in order to allow the optimal frustration and, consequently, the psychological growth of the client.]

3 [...optimal responsiveness, defined as the responsivity of the analyst that is therapeutically most relevant at any particular moment in the context of a particular patient and his
Tal postura exige que o terapeuta estabeleça uma relação flexível com o cliente e forneça respostas que facilitem o processo terapêutico, atendam às necessidades do cliente e contribuam para seu crescimento (Sousa, 2011).

Na década de 1990 Stiles et al. (1998) recuperam o conceito de responsividade a partir da perspectiva da Abordagem Centrada na Pessoa. Estes autores defendem que toda interação humana é "sistematicamente responsiva" (Stiles, Honos-Webb, \& Surko, 1998) e apresentam o conceito Responsabilidade Apropriada (ou Adequada), segundo a qual o terapeuta responsivo age de modo a alcançar um resultado desejado na experiência do cliente ou um padrão teórico exigido por uma abordagem terapêutica. O terapeuta deve estar atento e sensível ao seu cliente, reconhecendo déficits e recursos, de forma a intervir no momento adequado considerando suas especificidades.

De acordo com Stiles et al. (1998, p. 440) "O trabalho do terapeuta é monitorar a situação e escolher um tratamento apropriado para os problemas do cliente, seguir uma estratégia adequada à capacidade do cliente e intervir com técnicas apropriadas para o estado atual do cliente."

Hardy, Stiles, Barkham e Startup (1998) defendem que a responsividade apropriada deva ser julgada em relação ao objetivo (e não necessariamente em atender ao que o cliente solicita ou à teoria que embasa o tratamento) e levar em conta as restrições, o contexto e a história da relação. Quando pensada na interação terapeuta-cliente, a responsividade deve ser vista como a forma pela qual o terapeuta utiliza seu referencial teórico, suas técnicas e habilidades terapêuticas para responder ao cliente de forma sensível às suas necessidades, ainda que não necessariamente às solicitações imediatas deste.

illness. Empathy or vicarious introspection is the process by which the therapist comes to understand the patient by turning into his inner world. Optimal responsiveness on the other hand refers to a therapist at communicating his understanding to his patient.]

4 [The therapist's job is to monitor the situation and to choose a treatment that is appropriate for the client's problems, follow a strategy that is appropriate for the client's capacity, and intervene with techniques that are appropriate for the client's current state.] 
Stiles (2009) define a responsividade terapêutica como uma característica das interações humanas que envolve um ajuste contínuo de respostas, com base na natureza da situação interpessoal. A responsividade envolve as dimensões resposta e tempo, de forma que é possível dizer que uma pessoa responsiva é aquela que estabelece trocas com o outro, sabendo o que fazer e quando fazer. Com as respostas que ocorrem em sessão na interação terapeuta-cliente, a responsividade de saber o que fazer e quando fazer é descrita como capacidade de resposta apropriada (Hatcher, 2015; Stiles, 2009, 2013; Stiles et al., 1998, 1998).

Nota-se que, apesar de partir de diferentes perspectivas teóricas, todas as definições do termo responsividade enfatizam a sensibilidade do terapeuta às necessidades do cliente e sua resposta apropriada a essas necessidades, em direção à mudança terapêutica desejada.

\section{Conceituando a Responsividade a partir da Análise do Comportamento}

Para o analista do comportamento, uma resposta apropriada do terapeuta é aquela cuja função está atrelada a mudança do padrão comportamental que levou o cliente a um estado de sofrimento e busca por ajuda. Desta forma, mais do que a escolha da habilidade a ser usada (empatia, recomendação, interpretação, etc) e a discriminação do momento da interação em que esta habilidade é importante (tempo de sessão, etc), está em questão o responder contingente do terapeuta em relação ao cliente, levando em consideração a função dessa interação.

Quando o terapeuta age de maneira responsiva, as estratégias e habilidades terapêuticas que utiliza (empatia, solicitação de reflexão, recomendação, interpretação etc. - SiMCCIT Zamignani \& Meyer, 2011) podem ocorrer com intensidade e frequências variadas ao longo do processo psicoterápico, desde que respondam adequadamente às necessidades do caso clínico. Mesmo respostas que exerçam certa função aversiva, tais como o manter-se em silêncio, negar pedidos, confrontar, recomendar (Meyer, 2009;
Patterson \& Forgatch, 1985) podem se dar de maneira responsiva se a análise funcional contemplar a características imediatas e distais: (a) características pessoais, socioculturais, padrões de interação e repertório de entrada e atual do cliente; (b) momento da sessão terapêutica, do processo e da relação terapêutica estabelecida; (c) função imediata do comportamento do cliente na interação terapêutica; (d) operacionalização do comportamento em análise e (e) conceituação da queixa e objetivos terapêuticos definidos. Tal perspectiva extrapola aquela apontada por Hatcher (2015), que considera a empatia como componente central da responsividade.

Para a Análise do Comportamento, além da empatia, as respostas do terapeuta precisam estar em harmonia com a análise molar do caso para que de fato a relação seja responsiva e as respostas do cliente sejam consequenciadas de forma a se observar futuramente um efeito, quer seja no vínculo terapêutico de confiança mútua, quer seja nos relatos de meta e melhora. A análise molar permite a compreensão mais ampla e integrada do problema, considerando os aspectos históricos e atuais, de modo a evitar que o terapeuta responda em sessão apenas sob controle dos problemas semanais levantados pelo cliente. É a análise funcional molar, e não apenas molecular de um momento específico e de uma interação específica, que deve orientar o terapeuta quanto ao que fazer e quando fazer.

Dessa forma, guiado por uma formulação ampla do caso, as habilidades e técnicas utilizadas pelo terapeuta serão ativas e dirigidas a objetivos, sem que estejam excessivamente focadas em sintomas ou queixas pontuais ou pautadas em conselhos terapêuticos que tornem o cliente dependente das regras emitidas em sessão (Nery \& Fonseca, 2018).

Considerando os aspectos apontados nas referidas conceituações, pode-se definir responsividade na relação terapeuta-cliente a partir da perspectiva analítico-comportamental nos seguintes termos:

Responsividade terapêutica refere-se ao responder do terapeuta contíguo e contingente às necessidades do caso clínico e às variáveis de contexto da sessão terapêutica, tendo em vista as análises molecular e molar do caso. 


\section{Responsividade e a Pesquisa em Psicoterapia}

O trabalho de Eysenck, publicado em 1952 pode ser considerado um marco no incentivo da constante busca pela demonstração empírica dos resultados das psicoterapias, por questionar a eficácia dos processos terapêuticos e desencadear a busca por comprovação científica da efetividade da psicoterapia (Barlow, Boswell, \& Thompson-Hollands, 2013; Zamignani \& Meyer, 2014). Esse movimento se manteve e ocupou a atenção da APA (Associação Americana de Psicologia) na sua Divisão 12, denominada Sociedade de Psicologia Clínica.

Em 1993 a Divisão 12 da APA compôs uma força-tarefa de psicólogos com experiência em clínica e em pesquisa com os objetivos: a) definir e identificar tratamentos empiricamente validados (TEV); b) propor diretrizes de formação para futuros terapeutas e de treinamento contínuo para os profissionais em exercício; c) disseminar os TEV para os planos de saúde; e informar a população sobre a efetividade da psicoterapia (Chambless, Baker, Baucom et al., 1998). Nos últimos 10 anos, as práticas baseadas em evidências têm sido o principal alvo do desenvolvimento de pesquisas em psicoterapia (Kratochwill \& Stoiberm, 2002).

Tradicionalmente, os estudos sobre efetividade do processo terapêutico têm sido conduzidos por meio de ensaios clínicos randomizados (ECR). Neste tipo de investigação, são estudados grandes grupos de pacientes, distribuídos em grupos experimentais e grupos-controle - o primeiro deles recebendo o tratamento cuja efetividade deve ser estudada e o segundo recebendo um tratamento "placebo" (pacientes em grupo de espera, sem tratamento ou recebendo um tratamento que poderia ser considerado "inócuo", tais como uma conversa conduzida por um profissional não-qualificado) ou um tratamento já considerado padrão-ouro. A distribuição é feita randomicamente, ou seja, os participantes devem ter a mesma oportunidade de receber a intervenção proposta ou a intervenção controle. Para que se possa avaliar o impacto da intervenção proposta, a formação dos grupos deve ser o mais semelhante possível, de forma que a única diferença entre eles seja a própria intervenção.
Os resultados dos processos terapêuticos em ambos os grupos seriam medidos por meio de instrumentos padronizados de avaliação e comparados por meio de análises estatísticas e, se for demonstrado que o tratamento aplicado ao grupo experimental foi mais efetivo que o do grupo placebo ou equivalente ou superior ao tratamento padrão-ouro oferecido ao grupo-controle, então o tratamento experimental é considerado bem-sucedido. Tais delineamentos têm como requisito a padronização da intervenção por meio do desenvolvimento de protocolos que são descritos em manuais. Esses manuais precisam especificar as variáveis dependentes (os comportamentos-alvo, que em quase sua totalidade consistem em quadros diagnósticos descritos pelo DSM), as medidas padronizadas para a avaliação destes comportamentos-alvo, os mecanismos que produzem estes comportamentos e o plano de tratamento detalhado que deve atuar sobre tais mecanismos.

Stiles (2009), entretanto, aponta uma série de problemas que envolvem este método de produção de evidências, especialmente no que tange à responsividade. O primeiro deles diz respeito à própria concepção das variáveis que permeia os ECR. Nesse delineamento, o tratamento - que geralmente consiste em um protocolo com quantidade e sequência de sessões previamente determinadas - é considerado a Variável Independente (VI), ao passo que a mudança nos comportamentos-alvo é a variável dependente (VD). Com esta concepção, o estudo assume que a intervenção terapêutica teria uma função análoga àquela exercida pelo medicamento em um ECR nos estudos médicos. A questão é que, enquanto num estudo médico se investiga o efeito de uma única variável - uma substância que pode ser introduzida e retirada sobre um conjunto determinado de sintomas, na psicoterapia, a variável experimental é um combinado de procedimentos, que se distribui em uma série de encontros, constituídos por interações recíprocas. Num estudo médico os participantes são distribuídos randomicamente (controlando as variáveis estranhas) e se assume que cada grupo efetivamente recebe um tratamento diferente e que, portanto, as diferenças encontradas entre os grupos podem ser atribuídas com relativa segurança à substância introduzida. 
Já na psicoterapia, por mais bem descritos que sejam os manuais, é desejável que, perante diferentes tipos de clientes, o terapeuta implemente o tratamento de maneira diferente e se comporte de maneira diferente (mesmo que siga à risca o protocolo), sob pena do fracasso do tratamento se isso não ocorrer. É necessário um ajuste fino que possibilite o desenvolvimento do processo.

Ainda, segundo este mesmo autor, a responsividade é um fenômeno recíproco e assim como terapeutas conduziriam de maneira diferente os mesmos pacotes de intervenção perante clientes que apresentem estilos diferentes, o mesmo cliente poderia apresentar seu problema de maneira diferente na presença de terapeutas com estilos diferentes. Portanto, o estilo de interação do terapeuta responde ao estilo do cliente que por sua vez responde ao estilo do terapeuta, resultando em um complexo processo de determinação recíproca. Terapeutas são treinados a atentar e responder a indicações de melhora ou agravamento, mudanças no estado emocional, relatos de mudanças em relacionamentos ou em funcionamento no trabalho, evidências de se os problemas estão sendo superados ou resolvidos com sucesso. Terapeutas e clientes podem usar essas informações emergentes sobre os resultados das intervenções como sinalizadores para possíveis ajustes nas próximas intervenções, modificando sua atuação no processo terapêutico, visando tornar o tratamento mais eficaz. Desta maneira o tratamento ora assume o lugar de VI, ora de VD pois resultará da combinação de variáveis dessa interação recíproca. Ou seja, por mais desejável que seja a responsividade, ela introduz uma enorme variação no desenvolvimento do protocolo e, se há responsividade, os clientes nunca receberão o mesmo tratamento (Stiles, 2009).

Não apenas as variáveis Tratamento, Terapeuta, Cliente e Contexto dependem umas das outras, provocando os efeitos confundidores (supostamente avaliados nos ECR em psicoterapia), mas também essas VIs dependem, em parte, das VDs. A consideração do aspecto responsivo, indicando que cada medida efetuada nos estudos de resultado implicaria em um ajuste de rumo do procedimento, parece incompatível com a lógica experimental linear dos ECR (Stiles, 2009). A investigação de todas as combinações possíveis entre as variáveis parece inexequível. Assim, a intepretação dos dados referentes aos efeitos diferenciais da intervenção nos ECR em Psicoterapia é prejudicada.

Com base nestas constatações, Stiles (2009) defende a adoção de métodos qualitativos, narrativos e de estudo de caso como estratégias mais produtivas para investigar a psicoterapia e sua eficácia incluindo as maneiras pelas quais os clínicos usam o feedback de forma responsável (e responsiva) para melhorar o tratamento. Embora tais estratégias tenham menor poder com relação à validade externa, elas seriam mais sensíveis à complexidade das variáveis envolvidas.

O delineamento de sujeito único, por sua vez, tem sido o caminho por excelência para a produção de dados nos estudos conduzidos por analistas do comportamento. Portanto, a argumentação de Stiles dirige a investigação da psicoterapia para um campo muito familiar de domínio dos analistas do comportamento, para o qual têm sido desenvolvidos métodos sofisticados de investigação experimental e de controle de variáveis (vide Matos, 1990; Kratochwill \& Levin, 2014).

$\mathrm{Na}$ Análise do Comportamento o interesse pela pesquisa empírica esteve presente desde sua concepção ocupando um lugar privilegiado e reunindo os esforços de uma boa parcela dos profissionais (Tourinho, 2003). A Análise do Comportamento como ciência e o Behaviorismo Radical como filosofia defendem a visão contextualista. Isso significa que o comportamento só pode ser analisado e entendido no contexto, tanto pela perspectiva molar, quanto molecular. Desta forma, o contexto é definido tanto historicamente quanto situacionalmente. Reconhecer que os produtos da história são complexos, variáveis e idiossincráticos impõe ao terapeuta analítico comportamental que faça análises idiográficas (em oposição ao modelo nomotético) e intervenções igualmente individualizadas (Tourinho, 2003; Marçal, 2005).

Pesquisas em psicoterapia no âmbito da terapia analítico-comportamental começaram a ser desenvolvidas no Brasil, com intuito de identificar padrões de interação, classes de comportamentos do terapeuta e do cliente que pudessem estar relacionados ao sucesso terapêutico. Esses estudos, chamados pesquisas de processo, permitem a permitem a identificação de microprocessos e suas 
relações com a efetividade da intervenção, propiciando assim a construção de parâmetros mais fidedignos sobre a eficácia do método empregado (Marçal, 2005; Zamignani \& Meyer, 2014).

Nos estudos de processo em psicoterapia, entre as muitas variáveis da interação terapêutica, é possível investigar grau com que a díade terapeuta-cliente está engajada em um trabalho colaborativo e dirigido a um objetivo, o que é conhecido na literatura como Aliança terapêutica (Sousa, 2011). Tal aspecto leva em consideração a qualidade da Relação terapêutica, quer inclui o grau de envolvimento, a escuta e a flexibilidade do terapeuta perante as necessidades do cliente:

\begin{abstract}
Destacar a aliança como um processo colaborativo implica tanto a participação do terapeuta quanto do cliente na criação e manutenção de ações mútuas. Isso significa que conceber os clientes como ativos e colaboradores exige que os terapeutas escutem os clientes, privilegiem suas perspectivas, atendam às suas experiências subjetivas e ajustem ações terapêuticas flexíveis em resposta às suas necessidades. (Sousa, 2011, p. 5)
\end{abstract}

As revisões de pesquisa tendo por base a psicoterapia baseada em evidências mostram a importância da Relação Terapêutica afirmando que 1) a relação terapêutica proporciona contribuições substanciais e consistentes para o resultado da psicoterapia independentemente do tipo específico de tratamento; 2 ) é uma das razões pelas quais os clientes melhoram ou falham em melhorar, assim como nos métodos de tratamento tradicionais; 3 ) as práticas ou os guias terapêuticos deveriam especificar os comportamentos e qualidades do terapeuta que promoveriam o estabelecimento da relação terapêutica; 4) esforços para promover melhores práticas ou práticas baseadas em evidências que não incluam a relação terapêutica são seriamente incompletas e potencialmente equivocadas; 5) a adaptação da relação terapêutica às características particulares dos pacientes, além de um diagnóstico, podem melhorar a eficácia dos tratamento; 6) a relação terapêutica, junto com métodos de tratamento, características do paciente e as qualidades do terapeuta podem ser uma medida que determinam a eficácia ou ineficácia da psicoterapia (Norcross \& Wampold, 2011).
Nos estudos de processo, Stiles et al. (1998) defendem o desenvolvimento de métodos que não apenas levem em consideração, mas também permitam analisar o que denominam Responsividade Adequada como propriedade relevante. As pesquisas devem incluir tanto terapeuta quanto cliente como variáveis e reconhecer a interação entre eles. Deve-se tomar como medida do comportamento não apenas a frequência com que terapeuta ou cliente emitem uma ou outra categoria de resposta, mas também outras propriedades do comportamento, tais como a duração, a intensidade e os padrões de sequências de interações no andamento da sessão, visando detectar as relações entre as classes de respostas emitidas e seu respectivo contexto. Stiles et al. (1998) apontam algumas alternativas para o estudo da psicoterapia que levem em consideração a responsividade como propriedade relevante da interação:

- As análises devem incluir medidas de avaliação que incorporem julgamentos da responsividade, examinada por meio de qualificadores do comportamento - julgamentos positivos ou negativos sobre uma determinada intervenção, considerando a relação terapeuta-cliente, competências e habilidades terapêuticas. A aliança terapêutica também deveria ser avaliada, considerando o ajuste da ação do terapeuta ao contexto, ao cliente e vice-versa. Para o para o autor a aliança não é uma técnica terapêutica, e sim o reflexo do uso responsivo das técnicas que irão formar um composto de dimensões inter-relacionadas como vínculo, confiança, acordo sobre tarefas e objetivos. Os pesquisadores podem selecionar quais aspectos do comportamento são mais relevantes e julgar o valor por meio de versões de autorrelato ou versões avaliadoras de terceiros.

- Responsividade envolve necessariamente feedback: os efeitos de uma ação influenciam as ações subsequente. A maneira com que comportamentos do terapeuta e cliente se afetam repetidamente em espaços de tempo pode variar entre milissegundos a meses e os diálogos teriam que ser analisados como sistemas dinâmicos, não-lineares. Ou seja, abrangendo 
transformações que ocorrem ao longo da história de interações da díade. $\mathrm{O}$ aprimoramento de métodos estatísticos para análises de longas sequências de interação (podendo ser um contínuo de sessões) pode contribuir nesta direção.

- Abordagem qualitativas e narrativas: As narrativas do terapeuta e do cliente sobre uma sessão, sobre um evento da sessão ou sobre a relação terapêutica podem indicar aspectos significativos no processo de mudança do cliente e apontar para variáveis relevantes na sua construção, além de permitir acessar indicadores de responsividade adequada.

As consideração de Stiles et al. (1998) parecem estar em conformidade com as considerações da Associação Americana de Psicologia. Além das considerações sobre a relação terapêutica citadas anteriormente, a APA acrescenta a importância da elaboração e validação de manuais de tratamento que considerem variações que permitam certa flexibilidade e individualização do protocolo. Tal medida permitiria adaptar a intervenção às demandas e características dos clientes, sem dispensar a necessidade de descrição clara das variáveis de intervenção que possibilitem o treinamento para aqueles que os utilizarem. Quanto às características do delineamento de pesquisa, a Associação destaca a necessidade de programar avaliações por meio de diferentes métodos que evidenciem as medidas dos efeitos do procedimento, consistência dos efeitos, mecanismos causais do procedimento sobre as mudanças e a durabilidade dos resultados por meio de medições pós-tratamento (APA, 2006; Kratochwill \& Stoiber, 2002).

Kratochwill e Stoiber (2002) ao mencionarem sobre as principais necessidades de pesquisa destacam o desenvolvimento de medidas que possam ser usadas para quantificar o julgamento diagnóstico, avaliar o processo de intervenção e mensurar o progresso terapêutico a qualquer tempo. Tais medidas poderiam ser uma forma de suplementar as lacunas entre pesquisa e aplicação, com foco na promoção de competências comportamentais, emocionais e sociais, capazes de apontar aqueles componentes da intervenção responsáveis pela produção de uma mudança, além do desenvolvimento de hipóteses de porque as intervenções funcionam ou não funcionam (Kratochwill \& Stoiber, 2002; Wampold, Goodheart, \& Levant, 2007). Esses requisitos também podem compor as pesquisas e desenvolvimentos de intervenções na área clínica.

O conceito de Responsividade adquire suma importância na pesquisa clínica em análise do comportamento, ao explicitar o fenômeno interativo que dirige momento a momento o processo de mudança que ocorre na psicoterapia (Sousa, 2011) e dirigir a investigação clínica em conformidade com o método ideográfico. Desde o surgimento na análise experimental do comportamento, enquanto as demais ciências comportamentais substituíam o controle experimental pelo controle estatístico, Skinner e demais pesquisadores desenvolveram e aprimoraram os delineamentos de sujeito único (Matos, 1990), cujas características são mais sensíveis ao caráter individual, interacionista, processual e histórico do comportamento (Andery, 2010).

O fenômeno da Responsividade deve ser alvo de consideração mesmo no desenvolvimento de estudos com delineamentos de sujeito único, cuja manipulação de variáveis deve ser planejada de maneira peculiar, já que alguns tipos de delineamento podem se mostrar superiores em termo de sua sensibilidade à interação terapêutica responsiva. Por exemplo, enquanto um delineamento do tipo ABAB estabeleceria certa rigidez e linearidade no sequenciamento das fases do experimento, a randomização das fases em delineamentos multielementos pode introduzir certa flexibilidade, ao permitir alterações no delineamento previsto em função de eventuais mudanças no rumo da intervenção.

Luna (2012) salientou que fazer pesquisa, seja ela básica ou aplicada, impõe limites à atuação do pesquisador, como por exemplo, os concernentes ao método. $\mathrm{O}$ autor considera que o método utilizado por analistas do comportamento deve ser visto como uma lógica de raciocínio de que o pesquisador se vale para tomar decisões sobre o próximo passo. O método para o estudo em pesquisa aplicada vem sendo repensada e aprimorada de forma a ser possível estu dar fenômenos outrora apenas inferidos ou considerados como variáveis inespecíficas, como foi o caso da interação terapêutica. 
Este mesmo autor (1997) considera importante distinguir entre o pesquisar e o prestar serviços. Nas palavras dele:

Os critérios de relevância que presidem a formulação de um problema de pesquisa levam a esperar que a resposta a ser obtida ao final da pesquisa - constatada a correção metodológica - seja relevante para a comunidade científica por preencher uma lacuna importante no conhecimento disponível. Dessa forma, pesquisa é sempre um elo entre o pesquisador e a comunidade científica, razão pela qual a sua publicidade é elemento indispensável do processo de produção de conhecimento. Conhecimento não divulgado não é conhecimento, e seu produtor não é pesquisador. Nos projetos de prestação de serviços, o profissional (não necessariamente $o$ pesquisador) defronta-se com um interlocutor (indivíduo, grupo, comunidade) que apresenta um problema que, para maior facilidade de comunicação, identificarei aqui como "queixa". Cabe ao profissional identificá-la (ou levar o seu cliente a fazê-lo) e colocar sua competência a serviço do encaminhamento de soluções. Desta forma, um projeto de intervenção parte da "queixa" e tem como ponto de chegada, a sua solução. Se isto ocorrer, ter-se-á configurado uma bem-sucedida prestação de serviços." (Luna, 1997, p. 306)

Tal distinção leva à necessidade de se considerar de que modo uma pesquisa aplicada no âmbito da psicoterapia possa ser conduzida sem que se perca de vista os objetivos de uma prestação de serviços. Este autor discute o que chama de fetichização do procedimento, que sobrepõe as necessidades metodológicas da pesquisa às necessidades do caso clínico ou o que o autor refere como timing da intervenção:

Com exceção do caso de um terapeuta diretivo, em uma terapia diretiva, o timing do processo terapêutico está muito mais na dependência do cliente do que do terapeuta. Por outro lado, em condições gerais, o timing de uma pesquisa fica mais sob o controle do pesquisador do que do sujeito, até porque este pode vir a ser substituído. Nestas condições, resta ao terapeuta que pretenda produzir conhecimento enquanto intervindo na situação clínica, o desenvolvimento de um raciocínio metodológico ágil e criativo que permita, ao mesmo tempo, manter-se sob o controle das necessidades do cliente e daquelas da comunidade que julgará a sua produção. Como já foi dito antes, do ponto de vista epistemológico, o que interessa à comunidade é o conhecimento novo produzido, não o bem-estar do cliente; obviamente, do ponto de vista do cliente, a direção é inversa! (Luna, 1997, p. 311)

A problemática apontada por Luna (1997, 2012) indica uma questão pertinente ao tema do presente artigo. É compromisso ético do terapeuta priorizar o bem-estar do cliente e, no contexto da pesquisa, tal compromisso exige criatividade metodológica para eleger um delineamento que acolha de maneira responsiva às demandas do cliente ao mesmo tempo em que são respeitados os critérios científicos para o controle de variáveis que garanta a qualidade do dado produzido.

\section{Considerações Finais}

A responsividade, na díade cuidador-criança, é entendida como uma forma de atender às necessidades da criança para desenvolver sua autonomia e autoafirmação. Em outras palavras, é um comportamento sensível, por parte do adulto, que tem a finalidade de auxiliar o desenvolvimento da criança.

A tentativa de uma definição comportamental do termo responsividade enfatizou o responder habilidoso do terapeuta, de modo contíguo e contingente às necessidades do cliente, de forma a discriminar as variáveis de contexto e o controle de estímulos do cliente, tendo em vista a análise molar do caso em questão.

Tendo em vista que a responsividade é intrinsicamente relacionada à melhora dos problemas do cliente em um tratamento psicoterápico efetivo, é necessário considerá-la também ao se planejar um delineamento de pesquisa em clínica analítico-comportamental. 
O presente artigo convida o terapeuta/pesquisador a pensar em propostas metodológicas para a pesquisa em clínica analítico-comportamental que garantam não apenas o controle das variáveis que se propõem a medir, como também o estudo, e a medida apropriada de variáveis relacionadas à responsividade terapêutica.

\section{Referências}

Amaral, S. S. (2010). Efeitos da solicitação e da subsequente descrição dos relatos verbais de um terapeuta sobre seu desempenho em sessões posteriores. Dissertação de Mestrado. Pontifícia Universidade Católica de São Paulo.

Andery, M. A. P. A. (2010). Métodos de pesquisa em análise do comportamento. Psicologia USP, 21(2), 313-342. doi: 10.1590/S010365642010000200006

APA - American Psychological Association (2006). Presidential task force on evidence-based practice. Evidence-based practice in psychology. American Psychologist, 61, 271-285. doi: 10.1037/0003-066X.61.4.271

Bacal, H. A. (1985). Optimal responsiveness and the therapeutic process. In A. Goldberg (Ed.), Progress in self psychology, Vol. 1 ( pp. 202-227). New York: Guilford Press.

Bachelor, A., \& Horvath, A. (1999). The therapeutic relationship. In M. A. Hubble, B. L. Ducan, \& S. D. Miller (Eds.), The heart \& soul of change: what works in therapy (pp. 133-178). Washington D. C: American Psychologycal Association. doi:10.1037/11132-004

Barlow, D. H., Boswell, J. F., \& Thompson-Hollands, J. (2013). Eysenck, Strupp, and 50 years of psychotherapy research: A personal perspective. Psychotherapy, 50(1), 77-87. doi:10.1037/ a0031096

Bornstein, M. H. (1995). Form and function: Implications for studies of culture and human development. Culture \& Psychology, 1 (1), 123-137. doi: http://dx.doi. org/10.1177/1354067X9511009

Bowlby, J. (1984a). Apego. São Paulo: Martins Fontes.
Bowlby, J. (1984b). Separação. São Paulo: Martins Fontes.

Bowlby, J. (1988). Cuidados maternos e saúde mental. São Paulo: Martins Fontes.

Bretherton, I. (1992). The origins of attachment theory: John Bowlby and Mary Ainsworth. Developmental Psychology, 28, 759-775. doi:10.1037/0012-1649.28.5.759

Castonguay, L. G \& Beutler, L. E. (2006). Principles of therapeutics change that work. Oxford \& New York: Oxford University.

Chambless, D. L., Baker, M. J., Baucom, D. H., Beutler, L. E., Calhoun, K. S., Crits-Christoph, P., Daiuto, A., DeRubeis, R., Detweiler, J., Haaga, D. A. F., Bennett Johnson, S., McCurry, S., Mueser, K. T., Pope, K. S., Sanderson, W. C., Shoham, V., Stickle, T., Williams, D. A., \& Woody, S. R. (1998). Update on empirically validated therapies, II. The Clinical Psychologist, 51(1), 3-16.

Dicionário Infopédia da Língua Portuguesa. Porto: Porto Editora, 2003-2019. Recuperado em 11/05/2019 de: https://www.infopedia.pt/dicionarios/lingua-portuguesa/responsividade

Elliott, R., Bohart, A. C., Watson, J. C., \& Greenberg, L. S. (2011). Empathy. Psychotherapy, 48(1), 4349. doi:10.1037/a0022187

Eysenck, H. J. (1952). The effects of psychotherapy: an evaluation. Journal of Consulting Psychology, 16, 319-324. doi: 10.1037/h0063633

Follette, W. C.; Naugle, A. E.; Callaghan, G. M. (1996). A radical behavioral understanding of the therapeutic relationship in effecting change. Behavior Therapy, 27(4), 623-641. doi: 10.1016/ S0005-7894(96)80047-5

Hardy, G. E., Stiles, W. B., Barkham, M., \& Startup, M. (1998). Therapist responsiveness to client interpersonal styles during time-limited treatments for depression. Journal of Consulting and Clinical Psychology, 66(2), 304-312. doi:10.1037/0022-006x.66.2.304

Harwood, M. D. (2003). Effect of therapist process variables on treatment outcome for parentchild interaction therapy (PCIT). Masters Degree Dissertation. University of Florida.

Harwood, M. D., \& Eyberg, S. M. (2004). Therapist Verbal Behavior Early in Treatment: Relation to Successful Completion of Parent-Child Interaction Therapy. Journal of Clinical Child 
\& Adolescent Psychology, 33(3), 601-612. doi:10.1207/s15374424jccp3303_17

Hatcher, R. L. (2015). Interpersonal competencies: Responsiveness, technique, and training in psychotherapy. The American Psychologist, 70(8), 747-757. doi: 10.1037/a0039803

Kanamota, P. F. C., Bolsoni-Silva, A. T. \& Kanamota, J. S. V. (2016). A influência dos comportamentos de empatia e recomendação do terapeuta na interação terapeuta-cliente. Revista Interamericana de Psicologia/Interamerican Journal of Psychology, 50(3), 304-316. doi: 10.30849/rip/ijp.v50i3.24

Kivlighan, D. M., Angelone, E. O., \& Swafford, K. G. (1991). Live supervision in individual psychotherapy: effects on therapist's intention use and client's evaluation of session effect and working alliance. Professional Psychology: Research and Practice, 22(6), 489-495. doi:10.1037/07357028.22.6.489

Kohlenberg, R. J., \& Tsai, M. (2001). Psicoterapia analítica funcional: criando relações intensas e curativas. Santo André: Esetec Editores. (publicação original: 1991)

Kohut, H. (1989). Análisis del Self: el tratamiento psicoanalítico de los trastornos narcisistas de la personalidad. (M. A. Galmarini e M. Lucero, Trad.) Buenos Aires: Editorial Amorrortu. (Obra original publicada em 1971).

Kratochwill, T. R., \& Levin, J. R. (Eds.). (2014). Single-case intervention research: Statistical and methodological advances. Washington, DC, US: American Psychological Association. doi: 10.1037/14376-000

Kratochwill, T. R., \& Stoiber, K. C. (2002). Evidencebased interventions in school psychology: Conceptual foundations of the Procedural and Coding Manual of Division 16 and the Society for the Study of School Psychology Task Force. School Psychology Quarterly, 17(4), 341-389. doi: 10.1521/scpq.17.4.341.20872

Luna, S. V. (1997). O terapeuta é um cientista? Em: Banaco, R. A. (org.). Sobre Comportamento e Cognição: Aspectos Teóricos, Metodológicos e de Formação em Análise do Comportamento e Terapia Cognitivista (Vol. 1). Santo André: Arbytes.
Luna, S. V. (2012). Subsídios da prática da pesquisa para a prática clínica analítico-comportamental. In: Borges, N. B.; Cassas, F. A. (Org). Clínica analítico-comportamental aspectos teóricos e práticos (pp. 206-213). Porto Alegre: Artmed.

Maccoby, E. \& Martin, J. (1983). Socialization in the context of the family: Parent-child interaction. In P. H. Mussen, \& E. M. Hetherington (Eds.) Handbook of child psychology: Socialization personality and social development (Vol. 4, pp. 1-101). New York: Wiley.

MacDonald, K. B. (1998). Evolution, culture, and the five factor model. Journal of CrossCultural Psychology, 29(1), 119-149. doi: 10.1177/0022022198291007

Marçal, J. V. S. (2005). Estabelecendo objetivos na prática clínica: quais caminhos seguir? Revista Brasileira de Terapia Comportamental e Cognitiva, 7(2), 231-246.

Matos, M. A. (1990). Controle experimental e controle estatístico: a filosofia do caso único na pesquisa comportamental. Ciência e Cultura, 42, 585-592.

Meyer, S. B. \& Vermes, J. S. (2001). Relação terapêutica. In: Rangé, B. (Org.). Psicoterapias Cognitivo-Comportamentais: Um diálogo com a psiquiatria (pp. 101-110) Porto Alegre: Artmed.

Meyer, S. B. (2001). A Relação Terapêutica é o principal meio de intervenção terapêutica? In: Guilhardi, H. J; Madi, M. B. B. P; Scoz, M. C. Sobre Comportamento e Cognição: expondo a variabilidade (pp. 95-98). Santo André: ESETec.

Meyer, S. B. (2009). Análise de 'solicitação de informação' e 'recomendação' em banco de dados de terapias comportamentais. São Paulo, Tese de Livre-Docência (Departamento de Psicologia Clínica). Instituto de Psicologia da Universidade de São Paulo.

Meyer, S. B., Del Prette, G., Zamignani, D. R., Banaco, R. A., Neno, S., \& Tourinho, E. Z. (2010). Análise do comportamento e terapia analítico-comportamental. In: Emmanuel Z. T. e Sérgio V. L. (orgs), Análise do comportamento: investigações históricas, conceituais e aplicadas (pp. 153-174). São Paulo: Roca.

Nery, L. B., \& Fonseca, F. N. (2018). Análises funcionais moleculares e molares: um passo a passo. In: Ana Karina C. R.F, Flávia N. F. \& Lorena 
B. N. (Orgs.), Teoria e formulação de casos em análise comportamental clínica (pp. 22-54). Porto Alegre: Artmed.

Norcross, J. C. \& Wampold, B. E. (2011). Evidencebased therapy relationships: research conclusions and clinical practices. Psychotherapy, 48(1), 98-102. doi: 10.1037/a0022161

Orlinsky, D. E., Grawe, K., \& Parks, B. K. (1994). Process and outcome in psychotherapy: noch einmal. In A. E. Bergin \& S. L. Garfield (Eds.), Handbook of psychotherapy and behavior change (4th ed., pp. 270-376). New York: Wiley.

Oshiro, C. (2011). Delineamento experimental de caso único: a Psicoterapia Analítico Funcional com dois clientes difíceis. Tese de Doutorado. Universidade de São Paulo.

Oshiro, C., Kanter, J., \& Meyer, S. B. (2012). A single-case experimental demonstration of Functional Analytic Psychotherapy with two clients with severe interpersonal problems. International Journal of Behavioral Consultation and Therapy, 7(2-3), 111-116. doi: 10.1037/ h0100945

Papalia, D. E. \& Feldman, R. D. (2013). Desenvolvimento humano. Porto Alegre: ArtMed.

Patterson, G. R., \& Chamberlain, P. (1994). A Functional Analysis of Resistance During Parent Training Therapy. Clinical Psychology: Science and Practice, 1(1), 53-70. doi:10.1111/j.1468-2850.1994.tb00006.x

Patterson, G. R., \& Forgatch, M. S. (1985). Therapist behavior as a determinant for client noncompliance: A paradox for the behavior modifier. Journal of Consulting and Clinical Psychology, 53(6), 846-851. doi:10.1037/0022-006x.53.6.846

Pinto, M. G. A. (2007). Um estudo sobre relações entre o dizer e o fazer: algumas variáveis que operam no controle do planejamento de sessões terapêuticas. Dissertação de Mestrado. Pontifícia Universidade Católica de São Paulo.

Ribas, A. F. P. \& Moura, M. L. S. (2004). Responsividade Materna e Teoria do Apego: Uma Discussão Crítica do Papel de Estudos Transculturais. Psicologia: Reflexão e Crítica, 17 (3), 315-322. doi: 10.1590/S010279722004000300004
Ribas, A. F. P., Seidl de Moura, M. L. \& Ribas, R. C. Jr. (2003). Responsividade maternal: Levantamento bibliográfico e discussão conceitual. Psicologia: Reflexão \& Crítica, 16, 137-145. doi: 10.1590/S0102-79722003000100014.

Rocha, G. V. M. (2011). Empatia. In P. Gomide (Ed.), Comportamento moral: uma proposta para o desenvolvimento das virtudes (pp. 69-80). Curitiba: Juruá.

Santos, G. M., Santos, M. R. M., \& MarcheziniCunha, V. (2012). A escuta cautelosa nos encontros iniciais: a importância do clínico analítico-comportamental ficar sob controle das nuances do comportamento verbal. In N. B. Borges \& F. A. Cassas (Eds.), Clínica analíticocomportamental: aspectos teóricos e práticos (pp. 138-146). Porto Alegre: Artmed.

Silveira, F. F., Bolsoni-Silva, A. T., \& Meyer, S. B. (2010). Therapist's directive and nondirective behavior: analysis of their effects in a parent training group. International Journal of Behavioral Consultation and Therapy, 6(2), 124133. doi: $10.1037 / \mathrm{h} 0100900$

Silveira, J. M. (1997). A queixa clínica como condição para análise da interação terapeuta-cliente. Dissertação de mestrado. Universidade de São Paulo.

Silveira, J. M. (2000). Pesquisa da relação terapêutica em psicologia clínica comportamental. In C. E. Costa, J. C. Luzia, \& H. H. N. Sant'anna (Eds.), Primeiros passos em análise do comportamento e cognição (pp. 139-148). Santo André: Esetec Editores Associados.

Skinner, B. F. (2003). Ciência e Comportamento Humano. São Paulo: Martins Fontes, Publicação original de 1953.

Sousa, Z. C. R. (2011). Therapeutic Responsiveness as a moment-to-moment process of Alliance: Development of a conceptual-empirical model and an observational system. Tese de Doutorado. Universidade do Minho, Portugal.

Stiles, W. B. (1999). Signs and Voices in Psychotherapy. Psychotherapy Research, 9(1), 1-21. doi:10.1080/10503309912331332561

Stiles, W. B. (2009). Responsiveness as an obstacle for psychotherapy outcome research: It's worse than you think. Clinical Psychology: Science and Practice, 16(1), 86-91. doi: 10.1111/j.1468$-2850.2009 .01148 . x$ 
Stiles, W. B., Honos-Webb, L., \& Surko, M. (1998). Responsiveness in Psychotherapy. Clinical Psychology Science and Practice, 5(4), 439-458. doi: 10.1111/j.1468-2850.1998.tb00166.x

Tourinho, E. Z. (2003). A produção de conhecimento em psicologia: a análise do comportamento. Psicologia: ciência e profissão, 23(2), 3041. doi: 10.1590/S1414-98932003000200006.

Vermes, J. S., Zamignani, D. R., \& Kovac, R. (2007). A relação terapêutica no atendimento clínico em ambiente extra consultório. In: D. R. Zamignani; R. Kovac; J. S. Vermes (Orgs). A clínica de portas abertas: experiências e fundamentação do acompanhamento terapêutico e da prática clínica em ambiente extraconsultório (pp. 201-228). São Paulo: Editora Paradigma.

Wampold, B. E., Goodheart, C. D., \& Levant, R. F. (2007). Clarification and elaboration on evidence-based practice in psychology. American Psychologist, 62(6), 616-618. doi: 10.1037/0003066X62.6.616

Zamignani, D. R. \& Meyer, S. B. (2014). A pesquisa de processo em psicoterapia: Estudos a partir do instrumento SiMCCIT - Sistema Multidimensional para a Categorização de Comportamentos na Interação Terapêutica, (vol. 1). São Paulo, SP: Editora Paradigma.

Zamignani, D. R., \& Meyer, S. B. (2007). Comportamento verbal no contexto clínico: contribuições metodológicas a partir da análise do comportamento. Revista Brasileira de Terapia Comportamental e Cognitiva, 9(2), 241259. doi: 10.31505/rbtcc.v9i2.197

Zamignani, D. R., \& Meyer, S. B. (2011). Comportamentos verbais do terapeuta no Sistema Multidimensional para a Categorização de Comportamentos na Interação Terapêutica (SiMCCIT). Perspectivas em Análise do Comportamento, 2(1), 25-45. doi: 10.18761/ perspectivas.v2i1.47

\section{Informações do Artigo}

Histórico do artigo:

Submetido em: 14/12/2018

Primeira decisão editorial: 25/03/2019

Versão definitiva aceita em: 15/05/2019

Editor Associado: Denis Roberto Zamignani 